\title{
Tratamento cirúrgico da dissecção da aorta
}

Marcos Fassheber BERLINCK*, José Oscar Reis BRITO*, Salomon S. Ordinolla ROJAS*, Januário M. de SOUZA*, Sérgio de Almeida OLIVEIRA*

RBCCV 44205-108

BERLINCK, M. F.; BRITO, J. O. R.; RCJAS, S. S. O.; SOUZA, J. M.; OLIVEIRA, S. A. - Tratamento cirúrgico da dissecçăo da aorta. Rev. Bras. Cir. Cardiovasc., 5(1): 61-65, 1990.

RESUMO: Entre janeiro de 1979 e dezembro de 1989, foram realizadas 85 operaçōes para o tratamento da dissecçăo da aorta, sendo $\mathbf{5 0}$ na fase aguda e $\mathbf{3 5}$ na fase crónica. A mortalidade imediata (hospitalar) foi de $21,1 \%$ (18 pacientes), tendo como causa principal a síndrome de baixo débito cardiaco. Foi maior nos pacientes operados na fase aguda. Dentre quatro pacientes reoperados por recidiva ou dissecção em outro local, dois faleceram. Com relaçāo à morbidade, uma paciente, reoperada por aneurisma tóraco-abdominal, apresentou paraplegia no periodo de pós-operatório. O seguimento tardio mostrou boa evolução dos 63 sobreviventes.

DESCRITORES: dissecção da aorta, cirurgia.

\section{INTRODUÇÃO}

A dissecçăo da aorta é uma doença freqüente e grave, na sua evolução natural ${ }^{1,8-10}$.

O tratamento cirúrgico, iniciado há muitos anos ${ }^{1 .}$ 3. 8 , vem sofrendo modificaçōes técnicas ${ }^{2,4.7}$, sendo hoje realizado com bons resultados em um grande número de pacientes.

Neste trabalho, será analisada a experiência acumulada nos últimos 11 anos, para o tratamento da dissecção da aorta e suas complicaçōes, assim como as diferentes técnicas operatórias.

\section{CASUÍSTICA E MÉTODOS}

Entre janeiro de 1979 e dezembro de 1989, 81 pacientes foram operados para tratamento da dissecção da aorta, com um total de 85 operaçōes.

O diagnóstico da dissecção foi, quase sempre, confirmado pelo estudo cineangiográfico, ou através de tomografia computadorizada. Entretanto, em três dos pa- cientes com dissecção crônica de tipo II associada a acentuada insuficiência valvar aórtica, o diagnóstico da dissecçăo ocorreu durante a operaçăo.

Quatro pacientes foram operados duas vezes. Dois por recidiva e dois por dissecção em outros segmentos da aorta.

Sessenta e dois pacientes $(76,5 \%)$ eram do sexo masculino e $19(23,5 \%)$ do sexo feminino.

A idade variou entre 18 e 75 anos, com média de 45,7 anos. Foram operados 18 pacientes com mais de 60 anos.

Em 21 pacientes, havia doença associada, sendo: insuficiência coronária em 13, valvopatia aórtica em seis; coartação da aorta em um e persistência do canal arterial em um.

Dezessete pacientes haviam sido submetidos a cirurgia cardíaca prévia (Tabela 1), sendo sete para revascularizaçăo do miocárdio, seis para correçāo de lesão valvar aórtica e quatro que haviam sido submetidos a correção de dissecção da aorta anteriormente.

Trabalho realizado no Serviço de Cirurgia Cardiovascular do Prof. Dr. Sérgio Almeida de Oliveira - Hospital da Beneficéncia Portuguesa. Săo Paulo, SP. Brasil.

Recebido para publicaçăo em 10 de fevereiro de 1990.

Endereço para separatas: Marcos Fassheber Berlinck. Rua Maestro Cardim, 794. 01323 Săo Paulo, SP, Brasil. 
BERLINCK, M. F.; BRITO, J. O. R.; ROJAS, S. S. O.; SOUZA, J. M.; OLIVEIRA, S. A. - Tratamento cirúrgico da dissecçāo da aorta. Rev. Bras. Cir. Cardiovasc., 5(1): 61-65, 1990.

TABELA 1

PACIENTES COM CIRURGIA CARDIACA PREVIA

Cirurgia prévia No de pacientes

Revascularização do miocárdio

Troca valvar aórtica

Dissecção da aorta

$7^{*}$

6

4

- Pacientes em que a dissecção da aorta ocorreu durante o pós-operatório imediato.

TABELA 2

DISTRIBUIÇÃO POR TIPOS

\begin{tabular}{lll}
\hline Tipos & $1-\|(N \cdot)$ & III (N.) \\
\hline Agudos & 42 & 2 \\
Crônicos & 33 & 8 \\
\hline Total & $75(88,2 \%)$ & $10(13,3 \%)$ \\
\hline
\end{tabular}

Em três pacientes, a dissecção da aorta ocorreu no período intra-operatório, todos eles submetidos a cirurgia para revascularização do miocárdio.

Cinqüenta operaçōes foram realizadas na fase aguda e $\mathbf{3 5}$ durante a fase crónica. Um paciente foi operado após ressuscitação cárdiorespiratória.

As dissecçōes eram dos tipos I e II em 75 (88,2\%) e do tipo III em $10(11,8 \%)$ pacientes, segundo a classificação de De Bakey ${ }^{1}$, sendo, no primeiro grupo, dissecçōes agudas em 42 e crônicas em 33 . No segundo grupo, dois pacientes foram operados durante a fase aguda e oito em fase crônica (Tabela 2 ).

Os pacientes com disseção dos tipos I e II de De Bakey, admitidos durante a fase aguda, foram operados em caráter de urgência, quando apresentavam sinais de falência ventricular esquerda por insuficiência aórtica aguda, tamponamento cardiaco, ou por isquemia persistente dos membros ou órgāos.

Nas dissecçōes agudas do tipo III, optamos pelo tratamento clínico, ficando a cirurgia reservada para os pacientes com rápida expansão da dissecção, com hemorragia pleural ou isquemia aguda de membros ou órgãos.

$\mathrm{Na}$ fase crônica, tanto para as dissecçōes tipos I e II, como para as do tipo III, indicamos a operação na presença de aneurisma da aorta ou por disfunção valvar aórtica.

As operaçōes foram realizadas com estabelecimento de circulaçăo extracorpórea com canulação de artéria femoral comum e átrio direito, hemodiluição total e hipotermia sistêmica de 15 a $18^{\circ} \mathrm{C}$ (temperatura esofágica).
Nos últimos quatro anos, temos utilizado com grande freqüência a parada circulatória total, especialmente para a realização das anastomoses distais, nos casos de dissecção do tipo I, ou nas dissecçōes do tipo III. Nas dissecçōes do tipo III, a drenagem venosa foi realizada através da colocação de uma cânula na veia cava inferior através da veia femoral. Quando inadequada, foi complementada com drenagem do tronco pulmonar. Proteção miocárdica foi obtida com infusão de solução cardioplégica nos óstios coronários nos casos de dissecçōes envolvendo a aorta ascendente e croça. Até 1987, utilizamos a solução cristalóide tipo St. Thomas e, desde então, a solução sangüínea, segundo fórmula sugerida por BUCKBERG ${ }^{4}$. A valva aórtica foi conservada na grandemaioria dos pacientes (41/48). Apenas em sete pacientes a valva aórtica foi substituída, sendo empregada valva biológica em seis (valva porcina) e uma prótese rígida em um (Omniscience). A aorta ascendente foi substituída por um tubo de Dacron Woven* de baixa porosidade, pré-coagulado. Em alguns casos, o tubo foi embebido em sangue heparinizado e levado à autoclave a $110^{\circ} \mathrm{C}$ durante cinco minutos. Em três pacientes, um tubo de pericárdio bovino preservado foi utilizado. Em sete pacientes, a substituição da aorta foi realizada com tubo valvulado pela técnica de Bental-De Bonno (seis pacientes) ${ }^{3}$, ou com ligadura dos óstios coronários e implante de pontes de veia safena em dois pacientes. Estes dois últimos pacientes foram operados em 1979.

Para casos de dissecçāo aguda do tipo III, a via de acesso foi a toracotomia póstero-lateral esquerda na altura do quarto espaço intercostal. A circulação extracorpórea foi estabelecida por canulação da artéria femoral e tronco pulmonar.

A correção foi realizada com circulação extracorpórea com canulaçāo dos vasos femorais e para circulatória após o resfriamento do paciente para $18^{\circ} \mathrm{C}$.

Após a abertura da aorta e identificaçăo da área comprometida, foi interposto tubo de Dacron da maneira já descrita para a correçāo dos tipos I ou II. Este procedimento foi realizado em dois pacientes. Usamos, em ambos os casos, tubo de Dacron de baixa porosidade ( $\mathrm{Ta}$ bela 3 ).

TABELA 3

TÉCNICA OPERATORIA/AGUDOS

Técnica

Ie II (N.)

Substituição da Ao associada com conservação da valva aórtica .................................................................. 41

Tubo valvulado + reimplante de coronárias

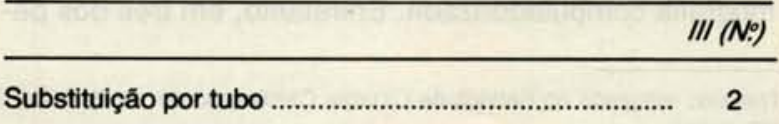

- Meadox Inc. U.S.A. 
BERLINCK, M. F.; BRITO, J. O. R.; ROJAS, S. S. O.; SOUZA, J. M.; OLIVEIRA, S. A. - Tratamento cirúrgico da dissecção

da aorta. Rev. Bras. Cir. Cardiovasc., 5(1): 61-65, 1990.

Nas dissecçōes crônicas dos tipos I e II, a aorta geralmente era bastante dilatada, formando aneurisma e com um maior grau de regurgitação aórtica.

A técnica operatória usada em 26 pacientes foi a descrita por Bental-De Bonno. Em sete, foi realizada a interposiçāo de um tubo na aorta ascendente, com troca valvar aórtica em três e comissurotomia aórtica em um paciente (Tabela 4).

TABELA 4

TÉCNICA OPERATORIANCRONICOS

\begin{tabular}{|c|c|}
\hline Técnica & Ie I| (N.) \\
\hline 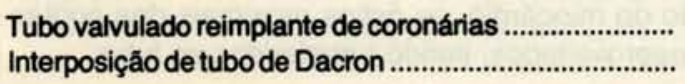 & $\begin{array}{r}26 \\
7\end{array}$ \\
\hline & III (N. \\
\hline 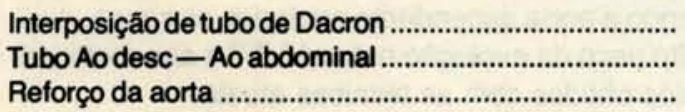 & $\begin{array}{l}6 \\
1 \\
1\end{array}$ \\
\hline
\end{tabular}

TABELA 5

MORTALIDADEJAGUDOS

\begin{tabular}{|c|c|}
\hline Etiologia & $\| / N$ \\
\hline $\begin{array}{l}\text { S.B.D.C. } \\
\text { A.V.C. }\end{array}$ & $\begin{array}{l}3 \\
1\end{array}$ \\
\hline Hemorragia (coagulopatia) & 3 \\
\hline Redissecção & 1 \\
\hline Infecção resporatória & 1 \\
\hline
\end{tabular}

\begin{tabular}{|c|c|}
\hline & III (N:) \\
\hline $\begin{array}{l}\text { Hemorragia digestiva } \\
\text { Coma (pré-operatório) }\end{array}$ & $\begin{array}{l}1 \\
1\end{array}$ \\
\hline
\end{tabular}

Foram operados oito pacientes em fase crônica com dissecção do tipo III. Em seis deles, a correçăo foi realizada com colocaçāo de tubo de Dacron; em um, foi anastomosado um tubo da aorta descendente à aorta abdominal e, em um paciente, foi realizado apenas um reforço na aorta (Tabela 4).

O revestimento do tubo com a parede da aorta dissecada foi realizado em muitos dos pacientes. Após a correção, quando há algum sangramento entre o tubo e o revestimento, colocamos um cateter (Jelco) entre as camadas e o conectamos a um aspirador. Com a pressão negativa gerada pela aspiração, ocorre o colapso do revestimento sobre o tubo, interrompendo o sangramento. Se persistir o sangramento após a neutralização da heparina, interpomos um pequeno tubo de PTFEE entre o saco aneurismático e o átrio direito.

\section{RESULTADOS}

A mortalidade imediata global (hospitalar) foi de $21,1 \%$ (18 pacientes), tendo sido $11(12,9 \%)$ no grupo de pacientes operados na fase aguda e sete na fase crônica (Tabela 5).

Os óbitos entre os pacientes operados durante a fase aguda por dissecçōes dos tipos I e II foram devidos a: síndrome de baixo débito cardíaco em três, hemorragia em três, acidente vascular cerebral em um, infecção pulmonar em um e redissecçăo em um. Entre os operados com dissecção do tipo III, um faleceu por hemorragia digestiva e 0 outro por lesão cerebral, estando este paciente em coma antes da operação (Tabela 5).

Para os pacientes operados durante a fase crônica com tipo I e tipo II, as causas de óbito foram: síndrome de baixo débito cardíaco em três, infarto agudo do miocárdio em um e hemorragia em um paciente (Tabela 6).

Já no grupo de pacientes operados por dissecção do tipo III, as causas foram: redissecção (um) e acidente vascular cerebral (um).

Entre os pacientes com dissecção da aorta e cirurgia cardíaca prévia, dois pacientes faleceram, sendo um no pós-operatório imediato, por síndrome de baixo débito e um em fase tardia por redissecção. Ambos haviam sido submetidos primariamente a cirurgia para substituição valvar aórtica.

As dissecçōes que ocorreram no período intra-operatório foram corrigidas com plastia da aorta e, deste grupo, uma paciente faleceu tardiamente, por redissecção.

Dos pacientes reoperados por redissecção da aorta, houve dois óbitos: uma paciente que havia sido submetida a correção de dissecção do tipo II desenvolveu, durante a gravidez, um aneurisma dissecante da croça, falecendo por insuficiência respiratória, e um paciente

TABELA 6

CAUSAS DE OBITOICAONICOS

Causas lell(N:)

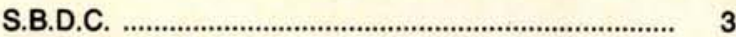

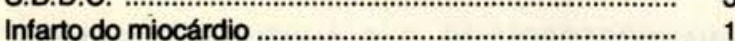

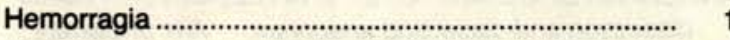

\begin{tabular}{|c|c|}
\hline & III (N.) \\
\hline $\begin{array}{l}\text { Dissecçāo da aorta ascendente } \\
\text { Coma (AVC) }\end{array}$ & $\begin{array}{l}1 \\
1\end{array}$ \\
\hline
\end{tabular}


BERLINCK, M. F.; BRITO, J. O. R.; ROJAS, S. S. O.; SOUZA, J. M.; OLIVEIRA, S. A. - Tratamento cirúrgico da dissecção da aorta. Rev. Bras. Cir. Cardiovasc., 5(1): 61-65, 1990.

que apresentou redissecção em outro segmento da aorta, vindo a falecer por baixo débito.

Uma paciente reoperada por ter desenvolvido aneurisma tóraco-abdominal apresentou paraplegia no pósoperatório.

\section{DISCUSSĀO}

Dos 17 pacientes submetidos a cirurgia cardiaca prévia, em alguns poucos pacientes a dissecção ocorreu no pós-operatório imediato, podendo este fato estar relacionado ao trauma operatório ${ }^{4}$.

Nos casos de processo de dissecção em pós-operatório tardio, provavelmente o mecanismo foi o mesmo do grupo de pacientes sem cirurgia cardíaca prévia.

Já o subgrupo com substituição valvar prévia talvez fosse formado por pacientes mais idosos e, por isso, com maior predisposiçăo à dissecçăo.

A fixaçăo proximal das camadas dissecadas da aorta utilizada na maioria dos casos operados em fase aguda às vezes permite a suspensão da valva aórtica, que fica com sua funcionalidade restabelecida.

A substituiçăo valvar aórtica, nestes pacientes operados na fase aguda, foi realizada quando havia doença primária da valva aórtica, ou quando a dissecçāo comprometia a raiz da aorta com destruição intensa dos seios de Valsalva e os óstios coronários.

A cola biológica* foi recurso utilizado em alguns casos, seja para fixar camadas da aorta ou reforçar a sutura dos óstios coronários, quando de sua transferência ao tubo.

Mais recentemente, temos usado o tubo corrugado de pericárdio bovino coberto de Dacron ${ }^{\star *}$, que consideramos bastante confortável de manusear, além de ser mais hemostático.

Em alguns pacientes com cirurgia prévia de revascularização do miocárdio, os óstios proximais das pontes foram reaproveitados, sendo transferidos ao tubo.

Em conclusāo, indicamos a cirurgia nos pacientes com diagnóstico de dissecção aguda dos tipos I e II envolvendo a aorta ascendente em todos os casos, devido ao alto risco da evolução natural ${ }^{3,8-10}$ e aos melhores resultados obtidos com as técnicas atuais.

Por outro lado, as dissecçōes do tipo III mostram risco cirúrgico aumentado e, por isso, temos restringido a indicação para aqueles casos com complicaçōes.

- Colagel - Cirumédica. São Paulo, SP.

* Labcor Laboratórios. Belo Horizonte, MG.

RBCCV 44205-108

BERLINCK, M. F.; BRITO, J. O. R.; ROJAS, S. S. O.; SOUZA, J. M.; OLIVEIRA, S. A. - Surgical treatment of the aortic dissection. Rev. Bras. Cir. Cardiovasc., 5(1): 61-65, 1990.

ABSTRACT: Between January 1979 and December 1989, eighty five operations were performed to treat aortic dissection, including fifty in the acute phase, and thirty five in a chronic phase. The Hospital mortality was $21.1 \%$ (eighteen patients) and low cardiac output was the major cause of death. The mortality was higher in the group of patients operated upon in the acute phase. Four patients were operated upon for redissection or dissection in other localization of the aorta, and all of them died. One patient developed paraplegy in the postoperative period. The late follow-up showed good evolution in the survivors group.

DESCRIPTORS: aortic dissection, surgery.

\section{REFERÊNCIAS BIBLIOGRÁFICAS}

1 ANAGNOSTOPOULOS, C. E. \& KITLE, C. F. - Aortic dissection and dissecting aneurysm. Am. J. Cardiol., 30: 263-273, 1972.

2 BACHET, J.; GIGOU, F.; LAURIAN, C.; BICAL, O.; GOUDOT, B.; GILMET, D. - Four-year clinical experience with the gelatin-resorcine-formol biologic glue in acute aortic dissection. J. Thorac. Cardiovasc. Surg., 83: 212-217, 1982.
3 BENTALL, H. \& De BONNO, A. - A tchnique for complete replacement of the ascending aorta. Torax, 23: 338-339, 1968.

4 BUCKBERG, G. D. - Strategies and logic of cardioplegia delivery to prevent, avoid and reverse ischemic and reperfusion damage. J. Thorac. Cardiovasc. Surg., 93: 127-139, 1987.

5 CABROL, C.; PAVIE, A.; GANDJBAKHCH, I. - Complete replacement of the ascending aorta with reimplantation of the coronary arteries: new surgical approach. J. Thorac. Cardiovasc. Surg., 81: 309-315, 1981. 
BERLINCK, M. F.; BRITO, J. O. R.; ROJAS, S. S. O.; SOUZA, J. M.; OLIVEIRA, S. A. - Tratamento cirúrgico da dissecção da aorta. Rev. Bras. Cir. Cardiovasc., 5(1): 61-65, 1990.

6 CABROL, C.; PAVIE, A.; GANDJBAKHCH, I. - Surgical treatment of ascending aorta pathology. J. Card. Surg., 3: 167-180, 1988.

7 COOLEY, D. A.; WALTER, W. F.; LIVEDAY, J. J.; OTT, D. A. - Ressection of aortic arch aneurysms: a comparison of hypothermic technics in 60 patients. Ann. Tho rac. Surg., 36: 19-28, 1983.
8 DAILY, P. O.; TRUEBLOOD, H. W.; STINSON, E. B.; SHUMWAY, N. E. - Management of acute aortic dissection. Ann. Thorac. Surg., 10: 237-247, 1970.

9 LARSON, E. W. \& EDWARDS, W. D. - Risk factors for aortic dissection: a necropsy study of 161 cases. Am. J. Cardiol., 53: 849-855, 1984.

10 ROBERTS, W. C. - Aortic dissections: anatomy, consequences and causes. Am. Heart J., 101 : 195-214, 1981. 